\title{
Análise do uso de combustível sólido para uso doméstico
}

\author{
Analysis of the use of solid fuel for domestic use \\ Análisis del uso de combustibles sólidos para uso doméstico
}

Recebido: 26/01/2022 | Revisado: 31/01/2022 | Aceito: 06/02/2022 | Publicado: 12/02/2022

\author{
Dennis Koji Fukace \\ ORCID: https://orcid.org/0000-0003-0858-6950 \\ Universidade Federal de São Carlos, Brasil \\ E-mail: dennisfukace@gmail.com \\ Diego Aleixo da Silva \\ ORCID: https://orcid.org/0000-0002-2552-7672 \\ Universidade Federal de São Carlos, Brasil \\ E-mail: diegoaleixo.florestal@gmail.com \\ Letícia Sant Anna Alesi \\ ORCID: https://orcid.org/0000-0001-6364-3028 \\ Universidade Federal de São Carlos, Brasil \\ E-mail: le_alesi1@hotmail.com \\ Elias Ricardo Durango Padilla \\ ORCID: https://orcid.org/0000-0001-5864-8828 \\ Universidade Estadual Paulista Júlio de Mesquita Filho, Brasil \\ E-mail: Erdurango22@gmail.com \\ Gabriela Bertoni Belini \\ ORCID: https://orcid.org/0000-0002-7231-694X \\ Universidade Federal de São Carlos, Brasil \\ E-mail: gabibelini@hotmail.com \\ Fabio Minoru Yamaji \\ ORCID: https://orcid.org/0000-0002-0908-8163 \\ Universidade Federal de São Carlos, Brasil \\ E-mail: fmyamaji@ufscar.br
}

\begin{abstract}
Resumo
Este trabalho teve como objetivo averiguar a qualidade de combustíveis sólidos para consumo doméstico. Os testes foram realizados a partir de seis tipos de materiais de origem arbórea do gênero Eucalyptus, sendo lenha, carvão vegetal e quatro tipos de briquete de carvão vegetal. Foram realizadas análises de umidade, análise química imediata, densidades, análise térmica e por microscopia eletrônica de varredura (MEV). Os resultados obtidos a partir destas análises se mostraram semelhantes ao da literatura. Os tratamentos provenientes de briquetes de carvão vegetal mostraram maior densidade energética aos demais, de todos os tratamentos o T4 foi o que apresentou melhor capacidade energética aos demais tratamentos sem correlação com os custos.
\end{abstract}

Palavras-chave: Biocombustível; Densidade energética; Poder calorífico.

\begin{abstract}
This work aimed to ascertain the quality of solid fuels for domestic consumption. The tests were carried out from six types of materials of tree origin of the genus Eucalyptus, being firewood, charcoal and four types of briquettes of charcoal. Analyzes of moisture, proximate analysis, densities, thermal analysis and by scanning electron microscopy (SEM) were performed. The results obtained from these analyzes were similar to those in the literature. Treatments from charcoal briquettes showed higher energy density than the others, of all treatments, the $\mathrm{T} 4$ was the one that presented better energy capacity to the other treatments without correlation with the costs
\end{abstract}

Keywords: Biofuel; Energy density; Calorific value.

\section{Resumen}

Este trabajo tuvo como objetivo investigar la calidad de los combustibles sólidos para el consumo doméstico. Las pruebas se realizaron utilizando seis tipos de materiales de origen arbóreo del género Eucalyptus, siendo leña, carbón vegetal y cuatro tipos de briquetas de carbón vegetal. Se realizaron análisis de humedad, análisis químico inmediato, densidades, análisis térmico y microscopía electrónica de barrido (SEM). Los resultados obtenidos de estos análisis fueron similares a la literatura. Los tratamientos a partir de briquetas de carbón vegetal presentaron mayor densidad energética que los demás, de todos los tratamientos el T4 fue el que presentó mejor capacidad energética a los demás tratamientos sin correlación con los costos.

Palabras clave: Biomasa; Bioenergía; Residuo. 


\section{Introdução}

O uso de combustível sólido pela humanidade teve início com o domínio do fogo utilizando a madeira como fonte de energia, primeiramente para realizar o aquecimento, proteção e cocção de alimento e, posteriormente, para a promoção de avanços tecnológicos (fins energéticos, indústria moveleira, fornos, etc.) que duram até os dias atuais (Brito, 2007), de modo que esta é responsável por suprir a demanda energética de aproximadamente 2,4 milhões de pessoas de vários países do mundo (Specht, 2012). A mesma autora ainda cita que, no Brasil, a lenha representa a segunda matriz energética do setor residencial e que seu uso é realidade bastante presente em áreas rurais (Areias et al, 2020a).

Nos setores domésticos e industriais da lenha, seu uso soma mais da metade do volume total de madeira consumida mundialmente para todas as finalidades (Areias et al, 2020b; Brito, 2007). De acordo com o Ministério de Minas e Energia (Brasil, 2015), o consumo total de lenha teve queda, no período de 2005 a 2014, de aproximadamente 10.000 mil toneladas, sendo que a sua transformação em carvão vegetal e o consumo final em residências correspondem por $31,1 \%$ e $24,7 \%$ do consumo total de lenha, respectivamente (Passos, 2015).

Esta transformação se torna eficiente uma vez que o carvão possui grande vantagem em relação à queima direta da madeira por possuir maior concentração de carbono e, portanto, maior poder calorífico superior (Barros, 2014; Resende et al., 2021). A briquetagem do carvão vegetal, que é a compactação ou prensagem de materiais particulados, resultando em um sólido com formas e dimensões controladas, pode surgir como uma alternativa para ampliar a oferta do carvão vegetal, com a vantagem de reduzir a pressão de exploração de florestas com espécies nativas (Lucena, 2008; Nakashima et al. 2017).

Por isso, além de diversas formas de se usar a madeira como combustível sólido, o reflorestamento de espécies exóticas, principalmente do gênero Eucalyptus, surge como opção para suprir a demanda de todos os setores. Com isso, este trabalho tem como objetivo analisar algumas propriedades químicas e físicas de diversos materiais combustíveis sólidos de Eucalyptus sp. para fins domésticos.

\section{Metodologia}

Os testes foram realizados no Laboratório de Biomassa e Bioenergia da Universidade Federal de São Carlos UFSCar, campus Sorocaba - SP. Os materiais utilizados foram cedidos pela Universidade e são da espécie arbórea Eucalyptus sp. (eucalipto) sendo: a lenha, carvão vegetal e quatro variedades de briquetes de carvão vegetal. Todo embasamento metodológico das análises foi feito de acordo com Nakashima et al. (2017).

\subsection{Descrição dos tratamentos}

Para a análise, foi estabelecido que os tratamentos fossem referentes aos diferentes tipos de materiais, tendo em T1 (lenha), T2 (carvão vegetal), T3 (briquete de carvão vegetal tipo cilíndrico) nacional, T4 (briquete de carvão vegetal tipo triangular) nacional, T5 (briquete de carvão vegetal tipo almofada) nacional, e T6 (briquete de carvão vegetal tipo almofada) estrangeira.

\subsection{Análise química imediata}

$\mathrm{Na}$ análise química imediata, foi determinado o teor de cinzas, teor de voláteis, e com isso o teor de carbono fixo, conforme a norma ASTM D1102/84. Para isso, na análise do teor de cinzas, foi pesado aproximadamente $1 \mathrm{~g}$ dos materiais seco em estufa a $105 \pm 2^{\circ} \mathrm{C}$, e inserido no cadinho previamente calcinado. Este e o material foram inseridos na mufla a 600 $\pm 10^{\circ} \mathrm{C}$ por 6 horas. Após a queima, o material (cinzas) foi pesado. Este procedimento foi realizado em triplicata para cada tratamento, para o cálculo do Teor de Cinzas (TC) foi utilizado a Equação (1). 


$$
\mathrm{TC}=\mathrm{mf} / \mathrm{mi} \times 100
$$

Onde: $\mathrm{TC}=$ teor de $\operatorname{cinzas}(\%) ; \mathrm{mi}=$ massa inicial $(\mathrm{g}) ; \mathrm{e} \mathrm{mf}=$ massa final $(\mathrm{g})$.

O teor de voláteis foi calculado conforme a norma ABNT NBR 8112/86. Inicialmente foi pesado aproximadamente $1 \mathrm{~g}$ do material seco em estufa a $105 \pm 2^{\circ} \mathrm{C}$, e inserido no cadinho previamente calcinado. O cadinho foi tampado com o material e inserido na mufla a $900 \pm 10^{\circ} \mathrm{C}$ por 7 minutos, pesando o material restante, este procedimento foi realizado em triplicata, para o cálculo do Teor de Voláteis (TV) foi utilizado a Equação (2).

$$
\mathrm{TV}=((\mathrm{mi}-\mathrm{mf}) / \mathrm{mi}) \times 100
$$

Onde: $\mathrm{TV}=$ teor de voláteis $(\%) ; \mathrm{mi}=$ massa inicial $(\mathrm{g}) ; \mathrm{e} \mathrm{mf}=$ massa final $(\mathrm{g})$.

Os valores obtidos foram aplicados à Equação (3) para a obtenção do Teor de Carbono fixo.

$$
\mathrm{TCF}=100-(\mathrm{TC}+\mathrm{TV})
$$

\subsection{Poder calorífico superior (PCS)}

Para a verificação do poder calorífico superior (PCS), foi introduzido aproximadamente $1 \mathrm{~g}$ de material isento de umidade no interior da bomba calorimétrica. A partir disto este foi inserido no calorímetro adiabático, marca IKA, modelo C200, e com base nos padrões da norma ASTM D5865/13, realizou-se o procedimento de determinação do poder calorífico superior dos materiais.

\subsection{Teor de umidade}

A obtenção do teor de umidade foi feita com base na norma ASTM D-3173, e os resultados foram descritos em percentual (\%) em relação à massa seca do material, conhecida como base seca.

Para a verificação do mesmo, os materiais foram enumerados e pesados para obter a massa inicial (g). Em seguida, foram levados para estufa a $105 \pm 2^{\circ} \mathrm{C}$ até atingir peso constante. $\mathrm{O}$ teor de umidade foi calculado conforme a Equação (4).

$$
\mathrm{TU}=((\mathrm{Mu}-\mathrm{Ms}) / \mathrm{Ms}) \times 100
$$

Sendo: $\mathrm{TU}=$ teor de umidade $(\%) ; \mathrm{Mu}=$ Massa úmida $(\mathrm{g})$; e Ms = Massa seca $(\mathrm{g})$.

\subsection{Densidade}

\subsubsection{Densidade básica}

A densidade básica é definida pela massa específica definida pela razão entre a massa seca (g) e o volume do material saturado $\left(\mathrm{cm}^{3}\right)$, determinada pela Equação (5). Esta metodologia foi utilizada para o T1, que foi o único que possibilitou a saturação do material, devido ao desprendimento de partículas nos demais tratamentos.

\subsubsection{Densidade aparente}

A densidade aparente dos materiais foi obtida pelos métodos de deslocamento de água e pela medição da geometria, com auxílio de um paquímetro, quando possível. Para a primeira metodologia, foi utilizado um béquer com água destilada em uma balança de precisão. O material foi envolvido em um papel filme, criando um sistema hidrofóbico e, com auxilio de uma garra de arame certificando que o material seja totalmente submerso. O volume da amostra foi então obtido ao se relacionar o valor da balança com a densidade da água. A obtenção do volume por meio da medição da geometria dos materiais pode ser feita quando a amostra apresentar forma geométrica prismática definida (T3 e T4), a averiguação do volume teve auxílio de um 
paquímetro digital, marca And A\&D Company, com precisão de 0,01 mm, neste caso o volume foi calculado medindo-se a altura, diâmetro e comprimento para o T3, e as arestas do prisma triangular, altura e comprimento para o T4.

Por fim, os materiais foram pesados em uma balança de precisão e medidos o teor de umidade, marca Shimadzu, modelo And MX-50. Sendo assim, foi possível medir a densidade aparente dos materiais a determinado teor de umidade pela Equação (5).

$$
\rho_{a_{i \%}}=\frac{M a}{V o l}
$$

Sendo: $\rho$ ai $\%=$ densidade aparente, em i\% de teor de umidade $(\mathrm{g} . \mathrm{cm}-3) ; \mathrm{Ma}=$ massa aparente do material $(\mathrm{g}) ; \mathrm{Vol}=$ volume do material $(\mathrm{cm} 3)$.

\subsubsection{Densidade energética}

A densidade energética dos materiais, um importante parâmetro para combustíveis sólidos, é calculada pela quantidade de energia armazenada em um determinado volume de material. Para isso, pegou-se o valor da densidade aparente de cada material e multiplicou seu valor pelo poder calorífico superior (PCS), conforme a equação (6), expressos em cal/cm³ . No caso do T1, cujo valor da densidade básica foi obtido, adicionou-se $12 \%$ do valor da densidade para acrescentar o respectivo teor de umidade.

$$
\rho_{e}=\rho_{a} x P C S
$$

Sendo: $\rho \mathrm{e}=$ densidade energética $\left(\mathrm{cal} / \mathrm{cm}^{3}\right) ; \rho \mathrm{a}=$ densidade aparente $\left(\mathrm{g} / \mathrm{cm}^{3}\right) ; \mathrm{PCS}=$ poder calorífico superior $(\mathrm{cal} / \mathrm{g})$.

\subsection{Análise das cinzas em microscopia eletrônica de varredura (MEV)}

As cinzas obtidas na análise química imediata de cada material foram analisadas com auxílio de um microscópio eletrônico de varredura (MEV), da marca Hitachi, modelo TM 3000 e auxílio do sistema de EDS (Energy Dispersive System), o que possibilitou a quantificação dos elementos químicos presentes em cada amostra.

\subsection{Análise estatística}

A análise estatística foi feita com o uso da linguagem computacional R (R Development Core Team, 2014), versão 3.4.3. Os resultados foram submetidos à análise de variância a fim de comparar os tratamentos. As médias foram então comparadas duas a duas por meio do teste Tukey (com 5\% de significância).

\section{Resultados e Discussão}

A análise de variancia apresentou diferença significativa entre os tratamentos justificando a aplicação do teste de Tukey (Tabela 1). Pode-se observar na tabela 1 que os tratamentos $\mathrm{t} 1$ e $\mathrm{t} 2$ não apresentaram diferença significativa entre si com relação aos teores de cinza o mesmo ocorreu para os tratamentos $\mathrm{t} 3$ e $\mathrm{t} 4$. Isso se da pelo fato de ambos serem matérias brutas não sofreram alterações físicas nem adição de compostos, que no caso dos outros tratamentos são a compactação de carvão vegetal juntamente com aglutinante para evitar desprendimento das partículas. 
Tabela 1. Teores encontrados na análise química imediata e teor de umidade

\begin{tabular}{cllll}
\hline Tratamento & TU $(\%)$ & TC $(\%)$ & TV $(\%)$ & TCF $(\%)$ \\
\hline T1 & $12,64 \mathrm{a}$ & $0,84 \mathrm{a}$ & $83,62 \mathrm{a}$ & $15,54 \mathrm{a}$ \\
$\mathrm{T} 2$ & $4,69 \mathrm{~b}$ & $0,10 \mathrm{a}$ & $32,65 \mathrm{~b}$ & $67,25 \mathrm{~b}$ \\
$\mathrm{~T} 3$ & $11,69 \mathrm{c}$ & $12,35 \mathrm{~b}$ & $23,11 \mathrm{c}$ & $64,54 \mathrm{~b}$ \\
T4 & $9,29 \mathrm{~d}$ & $12,17 \mathrm{~b}$ & $28,10 \mathrm{~d}$ & $59,73 \mathrm{c}$ \\
T5 & $9,95 \mathrm{e}$ & $19,01 \mathrm{c}$ & $27,96 \mathrm{~d}$ & $53,03 \mathrm{~d}$ \\
T6 & $10,73 \mathrm{f}$ & $16,20 \mathrm{~d}$ & $29,86 \mathrm{~d}$ & $53,94 \mathrm{~d}$ \\
\hline
\end{tabular}

Fonte: Autores (2021).

Para a quantidade de carbono fixo calculado para cada material, foi possível observar que os pares T2-T3 e T5-T6 não diferiram estatisticamente. O valor de T1 se mostrou superior a 10,4\% encontrado por Pincelli e colaboradores (2011). E T5 e T6 se apresentaram dentro dos encontrados por Dias Júnior e colaboradores (2014) com valores entre 46,00 - 52,00 \%. e T3 e T4 com resultados superiores a estes.

Os teores de compostos voláteis, para todos os tratamentos, apresentaram valores próximos aos encontrados na literatura. (Barros, 2014; Brito, 1978; Nones, 2014; Quirino, 1991; Trugilho, 2005; Santos, 2010), sendo que para os tratamentos que utilizaram o briquete de carvão vegetal apenas o T3 diferiu dos demais. Para o teor de umidade, todos os tratamentos tiveram diferenças significativas, podendo ser um fator importante para o potencial energético do biocombustível, uma vez que quanto menor seu teor, menor é o gasto de energia na forma de calor na fase inicial da queima (Santos, 2015).

$\mathrm{O}$ alto valor encontrado de teor de cinzas pelos tratamentos T3, T4, T5 e T6, que não apenas altera somente o potencial energético, mas também nos custos do processo. E, com isso, foi realizada a análise por microscopia eletrônica de varredura (MEV) para todos os tratamentos. E com o auxílio do EDS (Energy Dispersive System), foi possível a identificação e quantificação dos componentes químicos das cinzas. Estes se apresentam na forma de minerais que não fazem parte do processo de combustão (Tabela 2).

Tabela 2. Teores dos elementos químicos presentes nas cinzas dos materiais

\begin{tabular}{|c|c|c|c|c|c|c|}
\hline Tratamento & $\mathrm{Ca}(\%)$ & $\mathrm{K}(\%)$ & $\operatorname{Mg}(\%)$ & Mo (\%) & $\mathrm{Si}(\%)$ & $\mathrm{Al}(\%)$ \\
\hline $\mathrm{T} 1$ & 61,71 & 28,53 & 8,13 & 1,63 & - & - \\
\hline $\mathrm{T} 2$ & 44,41 & 36,02 & 7,54 & 12,03 & - & - \\
\hline $\mathrm{T} 3$ & - & - & 16,40 & - & 45,80 & 37,80 \\
\hline $\mathrm{T} 4$ & 82,93 & 12,28 & 4,69 & - & - & - \\
\hline T5 & - & - & 21,23 & - & 47,42 & 31,35 \\
\hline T6 & - & - & 29,91 & - & 44,57 & 25,52 \\
\hline
\end{tabular}

Fonte: Autores (2021).

Com isso, foi possível observar que para o T3, T5 e T6, a presença de Silício (Si) e Alumínio (Al) caracteriza elementos residuais do processo industrial que se juntaram aos briquetes durante seu processo de fabricação. A presença desses elementos pode inferir negativamente na sua capacidade energética. A ausência destes em T4 pode representar um erro metodológico na obtenção das amostras, retirando uma camada superficial das cinzas e, pelo fato destes elementos serem mais pesado e se depositarem na camada do fundo. 
Além disso, materiais de origem primária, T1 e T2 apresentaram apenas a presença de Cálcio (Ca), Potássio (K), Magnésio (Mg) e Molibdênio (Mo) elementos que, com suas ligações, formam elementos orgânicos das matérias. O alto valor de cinzas para o T4 e a ausência dos elementos residuais do processo industrial, não pôde ser explicado a partir desta análise.

A técnica de Microscopia Eletrônica de Varredura (MEV) foi utilizada para investigar a morfologia das cinzas dos tratamentos. No qual, podem-se observar morfologias estruturas residuais de elementos primários e também como estruturas residuais. Podendo ser quantificado, apenas, com o auxílio do EDS (Figura 1).

Figura 1. Imagens de Microscopia Eletrônica de Varredura (MEV) com ampliação de 800x para: A - T1; B - T2; C - T3; D T4; E-T5; e F - T6.

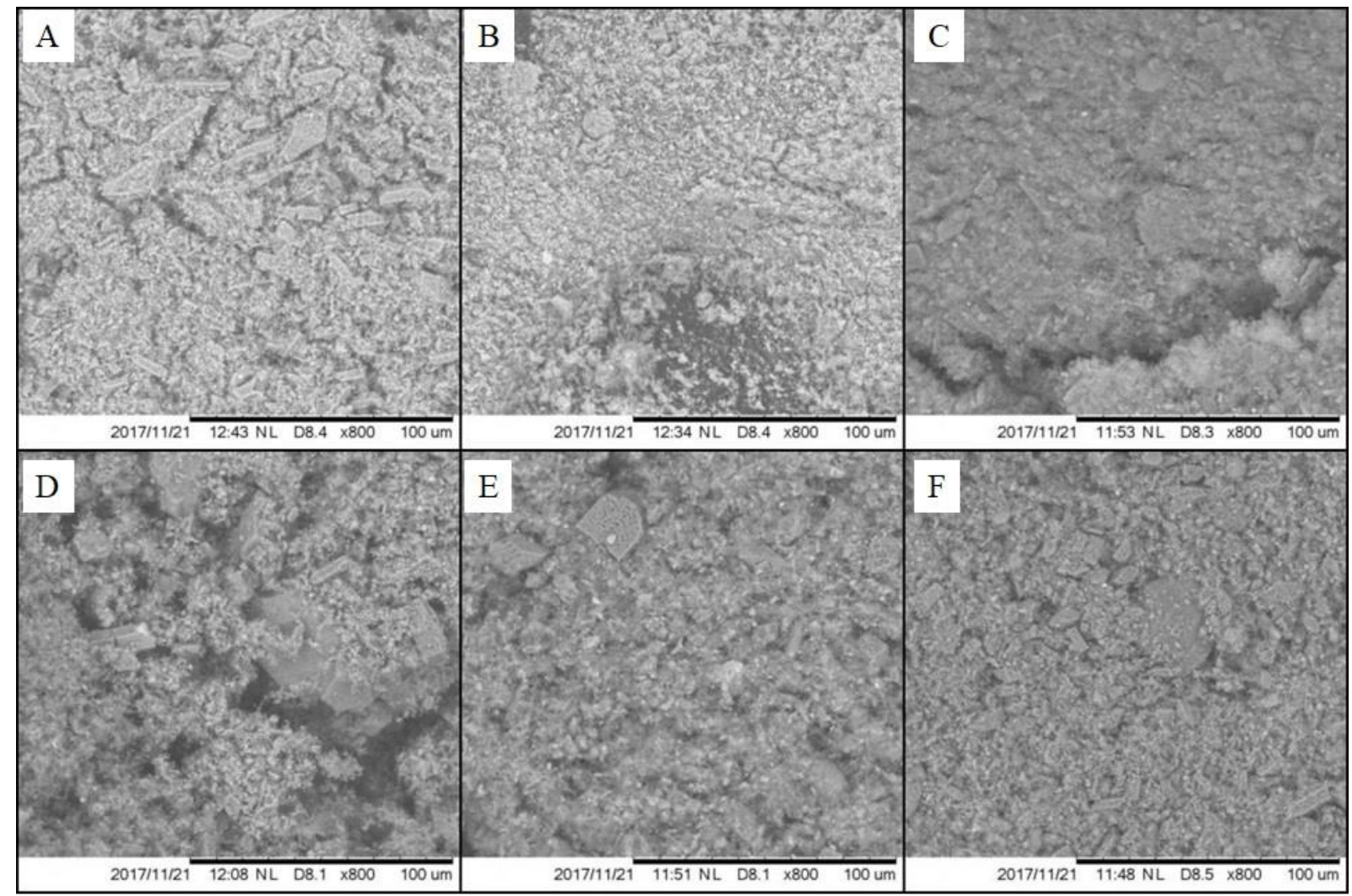

Fonte: Autores (2021).

Os resultados dos valores de teor de umidade, poder calorífico superior, densidade, densidade energética e custo dos materiais, estão descritos na Tabela 3. Foi possível perceber que o T1 se difere dos demais para o PCS, valor já observado por Barros (2014), em que encontrou valor próximo ao encontrado pelo T2 que utilizou do mesmo material em seus ensaios. 
Tabela 3. Poder calorífico superior (PCS), densidade, densidade energética e custo estimado dos materiais.

\begin{tabular}{|c|c|c|c|c|}
\hline Tratamento & PCS (Kcal/Kg) & Densidade $\left(\mathrm{g} / \mathrm{cm}^{3}\right)$ & $\begin{array}{c}\text { Densidade energética } \\
\left(\mathrm{cal} / \mathrm{cm}^{3}\right)\end{array}$ & Custo $(\mathrm{R} \$ / \mathrm{Kg})$ \\
\hline $\mathrm{T} 1$ & $4527,49 \mathrm{a}$ & $0,4708 \mathrm{a}$ & 2387,18 & 2,50 \\
\hline $\mathrm{T} 2$ & $7166,23 \mathrm{~b}$ & $0,4606 \mathrm{a}$ & 3300,79 & 4,75 \\
\hline $\mathrm{T} 3$ & $6130,98 \mathrm{c}$ & $0,7323 \mathrm{~b}$ & 4489,55 & 6,50 \\
\hline $\mathrm{T} 4$ & $6021,87 \mathrm{~d}$ & $0,9590 \mathrm{c}$ & 5774,90 & 7,00 \\
\hline T5 & $5306,64 \mathrm{e}$ & $0,8297 \mathrm{~d}$ & 4402,71 & 5,00 \\
\hline T6 & $5743,43 \mathrm{f}$ & $0,8394 \mathrm{~d}$ & 4820,79 & $3,00 *$ \\
\hline
\end{tabular}

*Valor obtido através da conversão cambial do Peso Argentino (ARS) para o nacional (BRL), na proporção de 1:5,33; BRL: ARS, na data de 06/12/2017. Fonte: Autores (2021).

Para o poder calorífico superior (PCS), todos os tratamentos se diferiram estatisticamente. Os valores obtidos nos testes foram equivalentes à literatura (Barros, 2014; Donato, 2015; Quirino, 1991; Pires, 2013; Teixeira et al., 2020; Wanderley, 2013). O mesmo se deu para os valores de densidade básica (T1) e aparente, onde apenas o T2 se mostrou levemente superior (Brand, 2015; Quirino, 1991; Ribeiro, 1993).

A partir dos valores obtidos para densidade energética, foi possível analisar que os briquetes de carvão vegetal se mostraram superiores aos da lenha e carvão vegetal, de forma que o T4 foi o que mostrou maior potencial de uso.

Os custos foram estimados a partir de pesquisa de mercado no varejo, ou disponíveis diretamente do produtor. De forma geral, os briquetes são mais caros que a lenha e o carvão vegetal, apenas para T6, que foi obtido a partir da conversão da moeda local, podendo fugir da realidade de pesquisa de mercado nacional.

\section{Conclusão}

Os valores de teor de carbono fixo (Tabela 1) e do poder calorífico superior (Tabela 3) se mostraram concomitantes, demonstrando a atribuição do aumento de carbono presente no material com seu respectivo poder calorífico.

Sugere-se realizar outra forma de análise ou repetir o processo para o alto teor de cinzas presentes no T4 para que seja possível sua atenuação e, consequentemente seu acréscimo no poder calorífico. Além disso, este tratamento se mostrou superior aos demais em relação à densidade energética.

Apesar das análises químicas, físicas e de custo, apenas o consumidor poderá definir que irá comprar, tendo influência de outros fatores além dos experimentais, tais como, marketing, promoções, preço de mercado, cultura, entre outros.

\section{Referências}

ABNT NBR 8112. (1986). Carvão vegetal - Análise Imediata. Rio de Janeiro: ABNT.

Areias, A. A.; Yamaji, F. M.; Cruz Junior, J. C. (2020a). Estudo de mercado sobre o uso de biomassa para queima no Estado de São Paulo, Brasil. Research, Society and Development, 9(11), e1429119566.

Areias, A. A., Cruz Júnior, J. C., Faria, L. C. de, \& Yamaji, F. M. (2020b). Uma abordagem logística e econômica para coordenar uma cadeia de suprimentos de biomassa, incluindo características de energia. Research, Society and Development, 9(8), e757986050.

ASTM D1102. (1984). Standard Test Method for Ash in Wood, ASTM International. West Conshohocken: ASTM International.

ASTM D3173. (2000). Standard Test Method for Moisture in the Analysis Sample of Coal and Coke. West Conshohocken: ASTM International.

ASTM D5865. (2013). Standard Test Method for Gross Calorific Value of Coal and Coke. West Conshohocken: ASTM International. 
Research, Society and Development, v. 11, n. 3, e5511326189, 2022

(CC BY 4.0) | ISSN 2525-3409 | DOI: http://dx.doi.org/10.33448/rsd-v11i3.26189

Barros, J. L. (2014) Caracterização de blendas e briquetes de carvões vegetal e mineral. Dissertação de Mestrado. Universidade Federal de São Carlos, Sorocaba, $125 \mathrm{f}$.

Brand, M. A.; Rodrigues, A. A.; De Oliveira, A. O.; Machado, M. S.; Zen, L. R. (2015). Qualidade do carvão vegetal para o consumo doméstico comercializado na região serrana sul de Santa Catarina. Revista árvore, 39(06), 1165-1173.

Brasil. Ministério de Minas e Energia. Empresa de Pesquisa e Energia (2015). Balanço Energético Nacional: relatório final. Rio de Janeiro,. 291 p.

Brito, J.O.; Barrichelo, L. E. G. (1978) Características do eucalipto como combustível: análise química imediata da madeira e da casca. IPEF, 16(1), 63-70.

Brito, J. O. (2007) O uso energético da madeira. Estudos Avançados, 21(59),185-193.

Dias Junior, A. F.; Andrade, A.M.; Costa Junior, D.S. (2014) Caracterização de briquetes produzidos com resíduos agroflorestais. Pesquisa florestal brasileira, 34(79), 225-234.

Donato, D. B.; Silva, C. M. S.; Magalhães, M. A.; Araújo Júnior, C. A.; Carneiro, A. C. O.; Vital, B. R. (2015) Propriedades de briquetes obtidos de finos de carvão vegetal. Ciência da Madeira, 6(2), 107-111, Viçosa.

Nakashima, G. T.; Adhmann, I. C. S.; Hansted, A. L. S.; Belini, G. B.; Waldman, W. R.; Yamaji, F. M. (2017) Materiais Lignocelulósicos: Caracterização e Produção de Briquetes. RVQ, 9(1), 150-162.

Nones, D. L.; Brand, M. A.; Cunha, A. B.; Carvalho, A. F.; Weise, S. M. K. (2015) Determinação das propriedades energéticas da madeira e do carvão vegetal produzido a partir de Eucalyptus benthamii. Floresta, 45(1), 57-64.

Passos, B. M.; Simioni, F.J.; Deboni, T.L.; Dalari, B.L.S.K. (2016) Características do consumo residencial de lenha e carvão vegetal. Floresta, 46(1), 21-29.

Pincelli, A. L. P. S. M. (2011) Características dos resíduos da colheita de madeira de eucalipto e pinus, submetidos ao tratamento térmico, com foco na aplicação energética. Tese Doutorado. Universidade de São Paulo, Escola Superior de Agricultura "Luiz de Queiroz", Piracicaba, 127f.

Pires, A. A. F. (2013) Resíduos lignocelulósicos para biocombustível sólido: caracterização e termogravimetria aplicada. Dissertação de mestrado. Universidade Federal de São Carlos, Sorocaba, 102 f.

Quirino, W. P., Brito, J. O. (1991) Características e índice de combustão de briquetes de carvão vegetal. Dissertação de Mestrado. Universidade de São Paulo, Escola Superior de Agricultura "Luiz de Queiroz", Piracicaba, 75 f.

R Core Team. (2017) R: A language and environment for statistical computing, R Foundation for Statistical Computing, http//www.R-project.org/

Resende, D. R., Araujo, E. da S., Lorenço, M. S., Zidanes, U. L. ., Bianchi, M. L. ., Trugilho, P. F. ., \& Mori, F. A. . (2021). Potencial energético dos resíduos do processamento de café combinados com finos de carvão vegetal. Research, Society and Development, 10(15), e326101523012.

Ribeiro, F. A.; Filho, J.Z. (1993) Variação da densidade básica da madeira em espécies/procedências de Eucalyptus spp. IPEF, 46(1), 76-85.

Santos, L. R. O. (2015) Análise da eficiência energética das biomassas utilizadas em fornos cerâmicos na região de Tatuí-SP. Dissertação de mestrado. Universidade Federal de São Carlos, Sorocaba, $69 \mathrm{f}$.

Santos, R. C dos. (2010) Parâmetros de qualidade da madeira e do carvão vegetal de clones de eucalipto. Dissertação de Mestrado. Universidade Federal de Lavras, Lavras, $175 \mathrm{f}$.

Specht, M. J. S. (2012) Uso de lenha como combustível doméstico: padrões, impactos e perspectivas futuras para conservação da florestal atlântica ao norte do rio São Francisco. Dissertação de Mestrado. Universidade Federal de Pernambuco, Refice, 75 f.

Trugilho, P. F.; Silva, J. R. M..; Mori, F. A..; Lima, J. T.; Mendes, L. M.; Mendes, L. F. B. (2005) Rendimentos e características do carvão vegetal em função da posição radial de amostragem em clones de Eucalyptus. Cerne, 11(2),178-186.

Teixeira, E. C.; Lima, A. P. L..; Lima, S. F..; Soratto, D. N.; Ramos, K. V.; Silva, W. G. (2020) Potencial energético da madeira de clones de eucalipto em diferentes espaçamentos. Research, Society and Development, 9(7), e02973733.

Wanderley, C. W. C. (2013) Caracterização de biomassa de toco e raíz de Eucalyptus urophylla S. T. Blake x Eucalyptus grandis W. Hill ex Maiden para geração de energia. Trabalho de conclusão de curso. Universidade Federal de São Carlos, Sorocaba, 17 f. 